\title{
Geometric algebra framework applied to circuits with non-sinusoidal voltages and currents
}

\author{
Jan L. Cieśliński ${ }^{(D)}$ and Cezary J. Walczyk ${ }^{\mathbb{D}}$ \\ Uniwersytet w Białymstoku, Wydział Fizyki, ul. Ciołkowskiego 1L, 15-245 Białystok, Poland; \\ j.cieslinski@uwb.edu.pl; c.walczyk@uwb.edu.pl \\ * Correspondence: j.cieslinski@uwb.edu.pl
}

Received: date; Accepted: date; Published: date

\begin{abstract}
We apply a well known technique of theoretical physics, known as Geometric Algebra or Clifford algebra, to linear electrical circuits with non-sinusoidal voltages and currents. We rederive from the first principles the Geometric Algebra approach to the apparent power decomposition. The important new point consists in a choice of a natural convenient basis in the Clifford vector space which simplifies considerably the presentation. Thus we are able to derive a number of general results which are missing in the former papers. In particular, a natural correspondence with the Current Physical Components approach is shown.
\end{abstract}

Keywords: nonsinusoidal voltages and currents; harmonics; power definitions; currents' physical components; Clifford algebras

\section{Introduction}

Complex numbers are with a long and deeply rooted tradition in description of alternating current (AC) circuits [1]. A sinusoidal function $f$, representing current or voltage, is represented by the so called phasor (complex function): $f(t)=\sqrt{2} F \cos (\Omega t+\alpha) \rightarrow \boldsymbol{F}(t)=\sqrt{2} F e^{j(\Omega t+\alpha)}$, where $j$ is the imaginary unit $\left(j^{2}=-1\right)$. The factor $\sqrt{2}$ is needed if we prefer to describe currents and voltages in terms of root mean square (RMS) values rather than in terms of amplitudes. Taking the real part of the phasor we recover the original sinusoidal function. The main reason for using this abstract (non-physical) space is a simple form of the Ohm Law for AC currents. For sinusoidal currents represented in the phasor space Ohm's Law has exactly the same form as for the direct current (DC) circuit $(\boldsymbol{U}=Z \boldsymbol{I})$ provided that instead of the real resistance one uses the complex impedance $Z$ which combines resistance, capacitance and inductance $(R, C$ and $L)$ in a well known way:

$$
Z=R+j\left(\Omega L-\frac{1}{\Omega C}\right), \quad Z=|Z| e^{j \varphi},
$$

where by $\varphi$ we denoted the phase shift between the current and voltage.

The complex impedance was first introduced by Kenelly [2]. In our article we prefer to use admittance (the reciprocal of impedance)

$$
Y=Z^{-1}=G+j B, \quad\left(Y=|Y| e^{-j \varphi}, \quad|Y|=\frac{1}{|Z|}\right),
$$

where the real part $G$ is known as the conductance and the imaginary part $B$ is called susceptance. Thus

$$
\boldsymbol{I}=Y \boldsymbol{U}
$$

and the current is computed as the real part of the product:

$$
i(t)=\operatorname{Re}\left((G+j B) \sqrt{2} U e^{j(\Omega t+\alpha)}\right)=\sqrt{2} U(G \cos (\Omega t+\alpha)-B \sin (\Omega t+\alpha)) .
$$

Here and in the rest of this paper we confine ourselves to single phase linear circuits (the nonlinear systems and three-phase systems will be considered elsewhere). 
Complex numbers yield a convenient interpretation of the active, reactive and apparent power in single phase linear circuits with a sinusoidal load:

$$
\boldsymbol{S}=\frac{1}{2} \boldsymbol{U} \boldsymbol{I}^{*}=U I^{*}=U U^{*} Y^{*}=|U|^{2} Y^{*}=U I e^{j \varphi}=U I \cos \varphi+j U I \sin \varphi
$$

where $U$ and $I$ are RMS values of the voltage and current, respectively. For our further purposes another representation of the apparent power will be useful:

$$
\boldsymbol{S}=\frac{1}{2} \boldsymbol{U} \boldsymbol{I}^{*}=U U^{*} Y^{*}=U^{2}(G-j B)
$$

Recently, another abstract mathematical structure has been applied to the classical problems of the electrical engineering. This is the Geometric Algebra (also known as the Clifford algebra), a quite popular and convenient tool in mathematical and theoretical physics (including electrodynamics) [3,4], which recent applications also in electrical engineering [5].

Since a dozen years, several authors try to make description of distoreted currents in terms of the Geometric Algebra. It seems that already the first attempt [6] was made in a good direction but later developments were of diffferent value and sometimes contain mistakes or too cumbersome developments [7-10]. As an example of a recent critique of the Geometric Algebra applications, see [11]. The recent paper by Montoya et al [12] seems to contain the most mature formulation of the problem.

Our paper has two main goals. First, we will derive from the first principles the Geometric Algebra formulation (in a form very close to the theory of Montoya [12]). The important new point consists in a choice of a natural convenient basis in the Clifford vector space. Second, we present a number of general results which are missing in the former papers (focusing on discussion of more or less representative examples). In particular, a natural correspondence with Czarnecki's current physical components approach $[13,14]$ is shown.

\section{Geometric Algebra}

Throughout this paper we consider non-sinusoidal currents in the form of finite sum of Fourier harmonics:

$$
\boldsymbol{u}=\sum_{k=1}^{N} U_{k} \tilde{\boldsymbol{c}}_{k}, \quad \tilde{\boldsymbol{c}}_{k}:=\sqrt{2} \cos \left(k \omega t+\alpha_{k}\right),
$$

Taking into account the possibility of currents' phase-shifts we have to consider $2 \mathrm{~N}$ dimensional vector space spanned by

$$
\tilde{\boldsymbol{c}}_{k}=\sqrt{2} \cos \left(k \omega t+\alpha_{k}\right), \quad \tilde{\boldsymbol{s}}_{k}=\sqrt{2} \sin \left(k \omega t+\alpha_{k}\right) .
$$

The voltage is a linear combination of $\tilde{\boldsymbol{c}}_{k}$ and the current, in general, can be a combination of all basis vectors.

The crucial point for the approach presented in this paper consists in treating products of functions: we use only the Clifford product (the Clifford product od two functions is completely different than the usual product of functions), for more details see the Appendix.

In this article, we intentionally mark periodic functions in bold, which means they have to be considered Clifford vectors wherever any product of these functions is involved.

Definition 1. The Clifford admittance $\boldsymbol{y}_{k}$ is defined as

$$
\boldsymbol{y}_{k}=G_{k}+\boldsymbol{j}_{k} B_{k}
$$

where $\boldsymbol{j}_{k}$ is a Clifford product of $\tilde{\boldsymbol{c}}_{k}$ and $\tilde{\boldsymbol{s}}_{k}$ :

$$
\boldsymbol{j}_{k}=\tilde{\boldsymbol{c}}_{k} \tilde{\boldsymbol{s}}_{k}
$$


The properties of $\boldsymbol{j}_{k}$ are very nice (for more details see the Appendix):

$$
\boldsymbol{j}_{k}^{2}=-1, \quad \boldsymbol{j}_{\mu} \boldsymbol{j}_{v}=\boldsymbol{j}_{v} \boldsymbol{j}_{\mu}
$$

These entities can be interpreted as commuting imaginary units related to every harmonics.

First of all, we are going to show that for sinusoidal currents our Clifford algebra approach yields the same results as the standard approach using complex phasors.

Theorem 2. Given a harmonic voltage $\boldsymbol{u}_{k}=U_{k} \tilde{\boldsymbol{c}}_{k}$ and a load with conductance $G_{k}$ and susceptance $B_{k}$, the current can be computed as the Clifford product of the voltage and the Clifford admittance:

$$
\boldsymbol{i}_{k}=\boldsymbol{y}_{k} \boldsymbol{u}_{k}
$$

The proof is straightforward:

$$
\boldsymbol{i}_{k}=\boldsymbol{y}_{k} \boldsymbol{u}_{k}=\left(G_{k}+\tilde{\boldsymbol{c}}_{k} \tilde{\boldsymbol{s}}_{k} B_{k}\right) U_{k} \tilde{\boldsymbol{c}}_{k}=U_{k}\left(G_{k} \tilde{\boldsymbol{c}}_{k}-B_{k} \tilde{\boldsymbol{s}}_{k}\right)
$$

The corresponding calculation using the usual complex phasors (compare (4) is a little bit more complicated, but, obviously, leads to the same result:

$$
\boldsymbol{I}_{k}=\operatorname{Re}\left(Y_{k} \boldsymbol{U}_{k}\right)=U_{k}\left(G_{k} \sqrt{2} \cos \left(\omega_{k} t+\alpha_{k}\right)-B_{k} \sqrt{2} \sin \left(\omega_{k} t+\alpha_{k}\right)\right)
$$

The important corollary is that a kind of a phasor-like structure is automatically built into the Clifford algebra structure and there is no need for a complexification of the Clifford algebra (as done, for instance, in [10]). In fact we already have $N$ commuting imaginary units $\boldsymbol{j}_{1}, \ldots, \boldsymbol{j}_{N}$ instead of the single complex imaginary unit $\boldsymbol{j}$.

The distorted (i.e., nonsinusoidal) case is treated in an analogous way due to the linearity of the problem:

$$
\boldsymbol{i}=\sum_{k=1}^{N} \boldsymbol{y}_{k} \boldsymbol{u}_{k}=\sum_{k=1}^{N}\left(G_{k}+\tilde{\boldsymbol{c}}_{k} \tilde{\boldsymbol{s}}_{k} B_{k}\right) \tilde{\boldsymbol{c}}_{k} U_{k}=\sum_{k=1}^{N}\left(G_{k} U_{k} \tilde{\boldsymbol{c}}_{k}-B_{k} U_{k} \tilde{\boldsymbol{s}}_{k}\right)
$$

Following Menti et al. [6] and Montoya et al. [12], we define geometric (Clifford) power as the Clifford product of $\boldsymbol{u}$ and $\boldsymbol{i}$.

Definition 3. Geometric power is defined as Clifford product of the voltage and the current (considered as Clifford vectors):

$$
\boldsymbol{M}=\boldsymbol{u} \boldsymbol{i}=\langle\boldsymbol{u} \mid \boldsymbol{i}\rangle+\boldsymbol{u} \wedge \boldsymbol{i}=\sum_{k=1}^{N} G_{k} U_{k}^{2}+\sum_{k, j=1}^{N} U_{j} U_{k}\left(G_{k} \tilde{\boldsymbol{c}}_{k} \wedge \tilde{\boldsymbol{c}}_{j}+B_{k} \tilde{\boldsymbol{c}}_{j} \wedge \tilde{\boldsymbol{s}}_{k}\right)
$$

It is worth to be noted that the above formula has a simplified form (as compared to earlier papers) due to the convenient choice of the basis $\tilde{\boldsymbol{c}}_{k}, \tilde{\boldsymbol{s}}_{k}(k=1, \ldots, N)$. It is a sum of orthogonal components. One can easily verify that the number of the orthogonal components $C_{N}$ is given by

$$
C_{N}=1+\frac{1}{2} N(N-1)+N^{2}
$$

The Clifford norm of $\boldsymbol{M}$ (the Clifford product of $\boldsymbol{M}$ and its reversion) equals $\boldsymbol{u}^{2} \boldsymbol{i}^{2}$ and this is the square of the apparent power value. By the standard Pythagorean-like rule it can be written as a sum of squares of the orthogonal components mentioned above.

Theorem 4. Clifford imaginary units depends only on the frequency (i.e., on k) and does not depend on the basis chosen (provided that it is also orthonormal and with the same orientation). In other words, $\tilde{\boldsymbol{c}}_{k} \tilde{\boldsymbol{s}}_{k}=\boldsymbol{c}_{k} \boldsymbol{s}_{k}$. 
Indeed, the transformation between the bases has the following form:

$$
\begin{aligned}
& \tilde{\boldsymbol{c}}_{k}=\boldsymbol{c}_{k} \cos \alpha_{k}-\boldsymbol{s}_{k} \sin \alpha_{k}=\left(\cos \alpha_{k}+\sin \alpha_{k} \boldsymbol{c}_{k} \boldsymbol{s}_{k}\right) \boldsymbol{c}_{k}=e^{\alpha_{k} \boldsymbol{j}_{k}} \boldsymbol{c}_{k}, \\
& \tilde{\boldsymbol{s}}_{k}=\boldsymbol{c}_{k} \sin \alpha_{k}+\boldsymbol{s}_{k} \cos \alpha_{k}=\left(\cos \alpha_{k}+\sin \alpha_{k} \boldsymbol{c}_{k} \boldsymbol{s}_{k}\right) \boldsymbol{s}_{k}=e^{\alpha_{k} \boldsymbol{j}_{k}} \boldsymbol{s}_{k}
\end{aligned}
$$

Then, we easily compute:

$$
\tilde{\boldsymbol{c}}_{k} \tilde{\boldsymbol{s}}_{k}=\boldsymbol{c}_{k} \boldsymbol{s}_{k} \cos ^{2} \alpha_{k}-\boldsymbol{s}_{k} \boldsymbol{c}_{k} \sin ^{2} \alpha_{k}+\left(\boldsymbol{c}_{k} \boldsymbol{c}_{k}-\boldsymbol{s}_{k} \boldsymbol{s}_{k}\right) \cos \alpha_{k} \sin \alpha_{k}=\boldsymbol{c}_{k} \boldsymbol{s}_{k}
$$

which ends the proof.

\section{Low dimensional special cases}

3.1. The case $N=1$.

The geometric power is computed easily as a sum of two terms:

$$
\boldsymbol{M}=\boldsymbol{u i}=G U^{2}+B U^{2} \tilde{\boldsymbol{c}}_{1} \wedge \tilde{\boldsymbol{s}}_{1}=G U^{2}+\boldsymbol{j}_{1} B U^{2},
$$

where we took into account, here and below, $\tilde{\boldsymbol{c}}_{1} \wedge \tilde{\boldsymbol{s}}_{1}=\tilde{\boldsymbol{c}}_{1} \tilde{\boldsymbol{s}}_{1}$ (because $\tilde{\boldsymbol{c}}_{1}$ and $\tilde{\boldsymbol{s}}_{1}$ anticommute). This result is, in principle, the same as obtained with the complex phasor approach (6). The difference in sign is either question of a convention or redefinition of the imaginary unit $(j \rightarrow-j)$.

3.2. The case $N=2$.

The geometric power is decomposed into the sum of $C_{2}=6$ terms (compare (17)):

$$
\begin{aligned}
\boldsymbol{M} & =\boldsymbol{u} \boldsymbol{i}=G_{1} U_{1}^{2}+G_{2} U_{2}^{2}+\left(G_{1}-G_{2}\right) U_{1} U_{2} \tilde{\boldsymbol{c}}_{1} \wedge \tilde{\boldsymbol{c}}_{2} \\
& +B_{1} U_{1}^{2} \tilde{\boldsymbol{c}}_{1} \wedge \tilde{\boldsymbol{s}}_{1}+B_{2} U_{2}^{2} \tilde{\boldsymbol{c}}_{2} \wedge \tilde{\boldsymbol{s}}_{2}+B_{1} U_{1} U_{2} \tilde{\boldsymbol{c}}_{2} \wedge \tilde{\boldsymbol{s}}_{1}+B_{2} U_{1} U_{2} \tilde{\boldsymbol{c}}_{1} \wedge \tilde{\boldsymbol{s}}_{2}
\end{aligned}
$$

where we took into account $\tilde{\boldsymbol{c}}_{2} \wedge \tilde{\boldsymbol{c}}_{1}=-\tilde{\boldsymbol{c}}_{1} \wedge \tilde{\boldsymbol{c}}_{2}$.

The scalar component $\left(G_{1} U_{1}^{2}+G_{2} U_{2}^{2}\right)$ is easily recognized as the active power. The next term, by $\tilde{\boldsymbol{c}}_{1} \wedge \tilde{\boldsymbol{c}}_{2}$, can be identified with the scattered power $\left(G_{1}-G_{2}\right) U_{1} U_{2}$. The remaining terms are related to the non-active power. The square of the apparent power can be decomposed as follows:

$$
\begin{aligned}
& \|\boldsymbol{u}\|^{2}\|\boldsymbol{i}\|^{2}=\left(G_{1} U_{1}^{2}+G_{2} U_{2}^{2}\right)^{2}+\left(\left(G_{1}-G_{2}\right) U_{1} U_{2}\right)^{2} \\
& +\left(B_{1} U_{1}^{2}\right)^{2}+\left(B_{2} U_{2}^{2}\right)^{2}+\left(B_{1} U_{1} U_{2}\right)^{2}+\left(B_{2} U_{1} U_{2}\right)^{2} .
\end{aligned}
$$

3.3. The case $N=3$.

In this case we have $C_{3}=13$ components:

$$
\begin{aligned}
\boldsymbol{M} & =\boldsymbol{u i}=G_{1} U_{1}^{2}+G_{2} U_{2}^{2}+G_{3} U_{3}^{2} \\
& +\left(G_{1}-G_{2}\right) U_{1} U_{2} \tilde{\boldsymbol{c}}_{1} \wedge \tilde{\boldsymbol{c}}_{2}+\left(G_{1}-G_{3}\right) U_{1} U_{3} \tilde{\boldsymbol{c}}_{1} \wedge \tilde{\boldsymbol{c}}_{3}+\left(G_{2}-G_{3}\right) U_{2} U_{3} \tilde{\boldsymbol{c}}_{2} \wedge \tilde{\boldsymbol{c}}_{3} \\
& +B_{1} U_{1}^{2} \tilde{\boldsymbol{c}}_{1} \wedge \tilde{\boldsymbol{s}}_{1}+B_{2} U_{2}^{2} \tilde{\boldsymbol{c}}_{2} \wedge \tilde{\boldsymbol{s}}_{2}+B_{3} U_{3}^{2} \tilde{\boldsymbol{c}}_{3} \wedge \tilde{\boldsymbol{s}}_{3} \\
& +B_{1} U_{1} U_{2} \tilde{\boldsymbol{c}}_{2} \wedge \tilde{\boldsymbol{s}}_{1}+B_{2} U_{2} U_{1} \tilde{\boldsymbol{c}}_{1} \wedge \tilde{\boldsymbol{s}}_{2}+B_{1} U_{1} U_{3} \tilde{\boldsymbol{c}}_{3} \wedge \tilde{\boldsymbol{s}}_{1} \\
& +B_{3} U_{3} U_{1} \tilde{\boldsymbol{c}}_{1} \wedge \tilde{\boldsymbol{s}}_{3}+B_{2} U_{2} U_{3} \tilde{\boldsymbol{c}}_{3} \wedge \tilde{\boldsymbol{s}}_{2}+B_{3} U_{3} U_{2} \tilde{\boldsymbol{c}}_{2} \wedge \tilde{\boldsymbol{s}}_{3}
\end{aligned}
$$


The scalar component $\left(G_{1} U_{1}^{2}+G_{2} U_{2}^{2}+G_{3} U_{3}^{2}\right)$ is, as always, the active power. The next three components form a vector sum of the orthogonal components of the scattered power. The square of its value is given by:

$$
\left(D_{s}\right)^{2}=\left(G_{1}-G_{2}\right)^{2} U_{1}^{2} U_{2}^{2}+\left(G_{1}-G_{3}\right)^{2} U_{1}^{2} U_{3}^{2}+\left(G_{2}-G_{3}\right)^{2} U_{2}^{2} U_{3}^{2} \text {. }
$$

We can see that the geometric (or Clifford) power is a sum of orthogonal componenets which can be interpreted within the standard CPC (curents' physical components) theory $[13,14]$. In fact, one can also make one to one correspondence on the level of currents. The active current is a Clifford vector parallel to the voltage. The scattered current is a component belonging to the subspace spanned by $\tilde{\boldsymbol{c}}_{1}, \ldots, \tilde{\boldsymbol{c}}_{N}$. The rest of the current is interpreted as non-active current.

\section{Conclusions}

In this paper we rederived the Geometric Algebra approach in application to the apparent power theory. The results are satisfactory and fully general. We would like to argue that the approach based on using the Clifford algebra is not more difficult than the standard complex phasor approach in the sinusoidal case but can be easily used also in the distorted case. Thus Clifford numbers can naturally replace complex numbers in the nonsinusoidal case.

Author Contributions: conceptualization, J.L.C.; methodology, J.L.C.; formal analysis, J.L.C. and C.J.W.; investigation, J.L.C. and C.J.W.; writing-original draft preparation, J.L.C.; writing-review and editing, J.L.C.

Funding: This research received no external funding.

Conflicts of Interest: The authors declare no conflict of interest.

\section{Appendix A. Geometric Algebra and its useful properties}

\section{Appendix A.1. Clifford product}

Given an $n$-dimensional vector space $V$ with a scalar product we can always construct the corresponding Clifford algebra by considering all "Clifford products". In this paper we consider the space of Fourier polynomials of order $N$, i.e., the space spanned by periodic functions (8). There is a natural scalar product in this space defined by the integral over the full period:

$$
\langle\boldsymbol{f} \mid \boldsymbol{g}\rangle=\int_{0}^{T} \boldsymbol{f}(t) \boldsymbol{g}(t) d t .
$$

where $T$ is the period. The voltage and currents are linear combinations of $\tilde{\boldsymbol{c}}_{k}$ and $\tilde{\boldsymbol{s}}_{k}(k=1, \ldots, N)$, and can be represented as vectors in the space of periodic functions with the period $T=2 \pi / \omega$.

Given a scalar product, the defining property of the Clifford product reads:

$$
f g+g f=2\langle f \mid g\rangle
$$

In other words, orthogonal Clifford vectors anticommute while parallel vectors commute. Clifford product can be always represented as a sum of the scalar product and the skew product:

$$
f g=\langle f \mid g\rangle+f \wedge g
$$

On the other hand, given a Clifford product, the skew (or wedge) product is defined easily as:

$$
f \wedge \boldsymbol{g}=\frac{1}{2}(\boldsymbol{f} \boldsymbol{g}-\boldsymbol{g} \boldsymbol{f})
$$

If $\langle\boldsymbol{f} \mid \boldsymbol{g}\rangle=0$, then, obviously, the Clifford product and the skew product coincide. 
Appendix A.2. Clifford products of basis vectors

The general rules of the Clifford product imply the following useful properties of the products of basis vectors (8):

$$
\begin{array}{lll}
\tilde{\boldsymbol{c}}_{k}^{2}=1, \quad \tilde{\boldsymbol{s}}_{k}^{2}=1, & \\
\tilde{\boldsymbol{c}}_{k} \tilde{\boldsymbol{s}}_{j}=-\tilde{\boldsymbol{s}}_{j} \tilde{\boldsymbol{c}}_{k}, \quad \tilde{\boldsymbol{c}}_{k} \tilde{\boldsymbol{s}}_{j}=\tilde{\boldsymbol{c}}_{k} \wedge \tilde{\boldsymbol{s}}_{j} & \\
\text { for } k \neq j: \quad \tilde{\boldsymbol{c}}_{k} \tilde{\boldsymbol{c}}_{j}=-\tilde{\boldsymbol{c}}_{j} \tilde{\boldsymbol{c}}_{k}, \quad \tilde{\boldsymbol{c}}_{k} \tilde{\boldsymbol{c}}_{j}=\tilde{\boldsymbol{c}}_{k} \wedge \tilde{\boldsymbol{c}}_{j}, \\
\text { for } k \neq j: \quad \tilde{\boldsymbol{s}}_{k} \tilde{\boldsymbol{s}}_{j}=-\tilde{\boldsymbol{s}}_{j} \tilde{\boldsymbol{s}}_{k}, \quad \tilde{\boldsymbol{s}}_{k} \tilde{\boldsymbol{s}}_{j}=\tilde{\boldsymbol{s}}_{k} \wedge \tilde{\boldsymbol{s}}_{j} .
\end{array}
$$

\section{Appendix A.3. Clifford product versus multiplication of periodic functions}

One has to remember that the Clifford product $\boldsymbol{f} \boldsymbol{g}$ cannot be identified with a usual product of two periodic functions (which is a periodic function as well, and can be decomposed with respect to the basis (8)). The product $\boldsymbol{f} \boldsymbol{g}$ is a construct belonging to a larger space known as a Clifford algebra.

For instance, let us consider the usual product of functions $\tilde{\boldsymbol{c}}_{1}$ (i.e, $\sqrt{2} \cos \left(\omega t+\alpha_{1}\right)$ ) and $\tilde{\boldsymbol{s}}_{1}$ (i.e, $\left.\sqrt{2} \sin \left(\omega t+\alpha_{1}\right)\right)$ :

$$
\sqrt{2} \cos \left(\omega t+\alpha_{1}\right) \sqrt{2} \sin \left(\omega t+\alpha_{1}\right)=\sin \left(2 \omega t+2 \alpha_{1}\right)
$$

which is a linear combination of $\tilde{\boldsymbol{c}}_{2}$ and $\tilde{\boldsymbol{s}}_{2}$ :

$$
\sin \left(2 \omega t+2 \alpha_{1}\right)=\lambda_{1} \cos \left(2 \omega t+\alpha_{2}\right)+\lambda_{2} \sin \left(2 \omega t+\alpha_{2}\right)
$$

where $\lambda_{1}=\sin \left(2 \alpha_{1}-\alpha_{2}\right)$ and $\lambda_{2}=\cos \left(2 \alpha_{1}-\alpha_{2}\right)$.

The Clifford product has no relation to the above computation. The Clifford product of $\tilde{\boldsymbol{c}}_{1}$ and $\tilde{\boldsymbol{s}}_{1}$ is another object outside the initial vector space $V$. In fact, this is a bivector $\tilde{\boldsymbol{c}}_{1} \tilde{\boldsymbol{s}}_{1}$. The Clifford product $\boldsymbol{c}_{1} \boldsymbol{s}_{1}$ cannot (and need not) be simplified. Certainly, $\tilde{\boldsymbol{c}}_{1} \tilde{\boldsymbol{s}}_{1} \neq \lambda_{1} \tilde{\boldsymbol{c}}_{2}+\lambda_{2} \tilde{\boldsymbol{s}}_{2}$.

\section{Appendix A.4. Commuting imaginary units}

An intriguing role in our approach is played by the elements $\boldsymbol{j}_{\mu} \equiv \tilde{\boldsymbol{c}}_{\mu} \tilde{\boldsymbol{s}}_{\mu}(\mu=1, \ldots, N)$, see (10). Let us show their most interesting properties.

$$
\tilde{j}_{\mu}^{2}=\tilde{\boldsymbol{c}}_{\mu} \tilde{\boldsymbol{s}}_{\mu} \tilde{\boldsymbol{c}}_{\mu} \tilde{\boldsymbol{s}}_{\mu}=-\tilde{\boldsymbol{c}}_{\mu} \tilde{\boldsymbol{c}}_{\mu} \tilde{\boldsymbol{s}}_{\mu} \tilde{\boldsymbol{s}}_{\mu}=-1
$$

where it was enough to use $\tilde{\boldsymbol{s}}_{\mu} \tilde{\boldsymbol{c}}_{\mu}=-\tilde{\boldsymbol{c}}_{\mu} \tilde{\boldsymbol{s}}_{\mu}$ and $\tilde{\boldsymbol{c}}_{\mu}^{2}=\tilde{\boldsymbol{s}}_{\mu}^{2}=1$. Then, for $\mu \neq v$ :

$$
\boldsymbol{j}_{\mu} \boldsymbol{j}_{v}=\tilde{\boldsymbol{c}}_{\mu} \tilde{\boldsymbol{s}}_{\mu} \tilde{\boldsymbol{c}}_{\nu} \tilde{\boldsymbol{s}}_{v}=-\tilde{\boldsymbol{c}}_{\mu} \tilde{\boldsymbol{c}}_{v} \tilde{\boldsymbol{s}}_{\mu} \tilde{\boldsymbol{s}}_{v}=-\tilde{\boldsymbol{c}}_{\nu} \tilde{\boldsymbol{c}}_{\mu} \tilde{\boldsymbol{s}}_{v} \tilde{\boldsymbol{s}}_{\mu}=\tilde{\boldsymbol{c}}_{\nu} \tilde{\boldsymbol{s}}_{\nu} \tilde{\boldsymbol{c}}_{\mu} \tilde{\boldsymbol{s}}_{\mu}=\boldsymbol{j}_{v} \boldsymbol{j}_{\mu}
$$

where we used few times commutation relations (A5). One can easily see that

$$
\boldsymbol{j}_{\mu} \tilde{\boldsymbol{c}}_{k}=\tilde{\boldsymbol{c}}_{k} \boldsymbol{j}_{\mu}, \quad \boldsymbol{j}_{\mu} \tilde{\boldsymbol{s}}_{k}=\tilde{\boldsymbol{s}}_{k} \boldsymbol{j}_{\mu}
$$

(imaginary unit $\boldsymbol{j}_{\mu}$ commutes with basis vectors $\tilde{\boldsymbol{c}}_{k}, \tilde{\boldsymbol{s}}_{k}$ for $k \neq \mu$ ). On the other hand,

$$
\boldsymbol{j}_{\mu} \tilde{\boldsymbol{c}}_{\mu}=-\tilde{\boldsymbol{c}}_{\mu} \boldsymbol{j}_{\mu}, \quad \boldsymbol{j}_{\mu} \tilde{\boldsymbol{s}}_{\mu}=-\tilde{\boldsymbol{s}}_{\mu} \boldsymbol{j}_{\mu}
$$

i.e., the imaginary unit $\boldsymbol{j}_{\mu}$ anticommutes with $\tilde{\boldsymbol{c}}_{\mu}$ and $\tilde{\boldsymbol{s}}_{\mu}$.

Thus we have here $N$ "imaginary units" $\boldsymbol{j}_{1}, \ldots \boldsymbol{j}_{N}$. It is tempting to see here a generalization of quaternions (where there are three imaginary units $(\boldsymbol{i}, \boldsymbol{j}, \boldsymbol{k})$ but one has to remember that our imaginary units commute $\left(\boldsymbol{j}_{\mu} \boldsymbol{j}_{v}=\boldsymbol{j}_{v} \boldsymbol{j}_{\mu}\right)$ while quaternion imaginary units anticommute (e.g., $\left.\boldsymbol{i} \boldsymbol{j}=-\boldsymbol{j} \boldsymbol{i}\right)$. A closer analogy can be made 
with the so-called scator algebra structure $[15,16]$, where any number of commuting imaginary units can appear (in the elliptic case).

\section{References}

1. C. P. Steinmetz. Complex quantities and their use in electrical engineering, Proceedings of the International Electrical Congress in Chicago, 21-25.08.1893, pp. 33-75. American Institute of Electrical Engineers, New York 1894.

2. A. E. Kenelly. Impedance, Transactions of the American Institute of Electrical Engineers 1893, vol. 10, 172-232.

3. D. Hestenes, G. Sobczyk: Clifford Algebra to Geometric Calculus: A Unified Language for Mathematics and Physics, D. Reidel Publ. Co., Dordrecht 1984.

4. J. Vaz, R. da Rocha. An Introduction to Clifford Algebras and Spinors, Oxford Univ. Press, Oxford 2016.

5. J. M. Chapell, S. P. Drake, C. L. Seidel, L. J. Gunn, A. Iqbal, A. Allison, D. Abbott. Geometric algebra for electrical and electronic engineers, Proceedings of the IEEE 2014102 (9), 1340-1363.

6. A. Menti, T. Zacharias, J. Milias-Argitis. Geometric algebra: a powerful tool for representing power under nonsinusoidal conditions, IEEE Transactions on Circuits and Systems I: Regular Papers 2007, it 54 (3), 601-609.

7. M. Castilla, J. C. Bravo, M. Ordóñez, J. C. Montaño. Clifford Theory: A Geometrical Interpretation of Multivectorial Apparent Power IEEE Transactions on Circuits and Systems I: Regular Papers 2008, it 55 (10), 3358-3367.

8. M. Castro-Núñez, R. Castro-Puche. Advantages of Geometric Algebra Over Complex Numbers in the Analysis of Networks With Nonsinusoidal Sources and Linear Loads, IEEE Transactions on Circuits and Systems I: Regular Papers 2012, it 59 (9), 2056-2064.

9. F. G. Montoya, R. Baños, A. Alcayde, F. M. Arrabal-Campos. A new approach to single-phase systems under sinusoidal and nonsinusoidal supply using geometric algebra, Electric Power Systems Research 2020, 189, 106605.

10. M. V. Castilla, F. Martin. A Powerful Tool for Optimal Control of Energy Systems in Sustainable Buildings: Distortion Power Bivector, Energies 2021, 14, 2177.

11. L. S. Czarnecki, M. Almousa. What is Wrong with the Paper "The IEEE Standard 1459, the CPC Power Theory and Geometric Algebra in Circuits with Nonsinusoidal Sources and Linear Loads”?, Przeglad Elektrotechniczny 2020, 96 (7), 1-7.

12. F. G. Montoya, R. Baños, A. Alcayde, F. M. Arrabal-Campos, J. Roldán-Pérez. Vector Geometric Algebra in Power Systems: An Updated Formulation of Apparent Power under Non-Sinusoidal Conditions, Mathematics 2021, 9 , 1295.

13. L. S. Czarnecki. Currents' physical components (CPC) in circuits with nonsinusoidal voltages and currents. Part 1: Single phase linear circuits. Electrical Power Quality and Utilisation Journal 2005, 11 (2), 3-14.

14. L. S. Czarnecki. Currents' Physical Components (CPC) - based Power Theory. A Review. Part I: Power Properties of Electrical Circuits and Systems, Przeglad Elektrotechniczny 2019, 95 (10), 1-11.

15. A. Kobus, J. L. Cieśliński. Geometric and Differential Features of Scators as Induced by Fundamental Embedding, Symmetry 2020, 12 (11), 1880.

16. J. L. Cieśliński, A. Kobus. Group Structure and Geometric Interpretation of the Embedded Scator Space, Symmetry 2021, 13 (8), 1504. 